\title{
FONTES E DISTRIBUIÇÃO DA MATÉRIA ORGÂNICA SEDIMENTAR NA PORÇÃO NOROESTE DA BAÍA DE VITÓRIA, ES.
}

\author{
LEHRBACK, B. D. ${ }^{1, *}$; NETO, R. R. ${ }^{1}$; BARROSO, G. F. ${ }^{1}$ \& BERNARDES, M. C. ${ }^{2}$ \\ 1. Universidade Federal do Espirito Santo, ES, Brasil. \\ 2. Universidade Federal Fluminense, RJ, Brasil. \\ ${ }^{*}$ Corresponding author: bethania.dalcol@gmail.com
}

\begin{abstract}
Lehrback, B. D.; Neto, R. R.; Barroso, G. F. \& Bernardes, M. C., (2016). Fontes e distribuição da matéria orgânica sedimentar na porção noroeste da baía de Vitória, ES. Braz. J. Aquat. Sci. Technol. 20(1). elSSN 1983-9057. DOI: $10.14210 /$ bjast.v20n1. The sources and distribution of the organic matter $(\mathrm{MO})$ were evaluated in sediments of the northwest region of the Vitória Bay, Espírito Santo State, Southeast Brazil. The bay showed a predominance of terrestrial sources in relation to other sources (aquatic and anthropogenic), mainly in the northern portion of the bay nearby the mangroves. This can be evidenced by the presence of compounds like the triterpenoids $\beta$-amyrin (the highest concentration; $87.2 \mu \mathrm{g} . \mathrm{g}^{-1}$ ), besides lupeol, germanicol, taraxerol and $\alpha$-amyrin, and the sterols $\beta$-sitosterol (most abundant sterol; $7.8 \mu$ g. $g^{-1}$ ), stigmasterol and campesterol. The distribution of fatty acids, n-alkane, Terrestrial Aquatic Ratio (TAR), Carbon Preference Index $(\mathrm{CPI})$ and the ratio $\mathrm{C} / \mathrm{N}$, also characterized the terrestrial input. Although the Vitória Bay and region are characterized by intense human activities, the molecular composition of the sediments indicated a intermediate level of fecal pollution, with coprostanol concentration between $0.2 \mu \mathrm{g} \cdot \mathrm{g}^{-1}$ and $5.2 \mu \mathrm{g} \cdot \mathrm{g}^{-1}$, and $5 \beta /(5 \alpha+5 \beta)$ stanol index around 0.6. Markers of fossil oil were also found (ex. hopanoids with configuration $17 \alpha(\mathrm{H}), 21 \beta(\mathrm{H}))$, more concentrated in the southern part of the studied region, where the urban activity is more intense. Cholesterol showed maximum concentration of $4.7 \mu \mathrm{g} . \mathrm{g}^{-1}$.
\end{abstract}

Keyworks: Estuary, Lipids, Biomarkers.

\section{INTRODUÇÃO}

Estuários são ecossistemas costeiros localizados na foz de rios, que apresentam elevada importância para os processos biogeoquímicos da matéria orgânica (MO) e exportação de material para ecossistemas marinhos. A origem da $\mathrm{MO}$ nestes sistemas apresenta-se bastante complexa, podendo prover de fontes autóctones e alóctones (McCallister et al., 2006; Volkman et al., 2007; Kristensen et al., 2008). Os estuários tropicais e subtropicais são associados aos ecossistemas de manguezais, que se destacam por uma distinta vegetação adaptada aos pronunciados gradientes físico-químicos (Elliott \& McLusky, 2002; Moberg \& Rönnbäck, 2003). Ecossistemas manguezais se caracterizam por possuírem elevada produtividade de carbono orgânico, dessa forma, apresentam um importante papel no ciclo do carbono para ambientes estuarinos, através da exportação deste material para estes ambientes (Killops \& Frewin, 1994; Dodd et al., 1998; Versteegh et al., 2004; Koch et al., 2005).

A MO representa um importante componente para a cadeia alimentar de ecossistemas estuarinos. Sendo assim, a investigação de aspectos qualitativos e dinâmica da MO estuarina, sobretudo quanto às alterações diagenéticas, consumo, deposição e exportação é de grande relevância para melhor compreensão do ciclo global do carbono (Hopkinson et al.,
1998; Jaffé et al., 2006). Além de processos naturais de natureza física, química e biológica que influenciam a quantidade e composição da $\mathrm{MO}$, esta também é fortemente influenciada por ações antrópicas ao longo do gradiente fluvio-estuarino, tais como: aporte de efluentes domésticos ou industriais tratados ou in natura, desmatamento, fontes difusas de fertilizantes e pesticidas utilizados na agricultura, represamento fluvial por barragens, entre outros (Jaffé et al., 1995). A caracterização da MO pode ser realizada através da utilização de lipídeos como marcadores moleculares, devido a relativa estabilidade destes compostos orgânicos em relação a outros, tais como proteínas e aminoácidos (Jaffé et al., 2001; Medeiros \& Bícego, 2004; Otto \& Simpson, 2005). Por esses motivos, o conhecimento acerca da composição molecular lipídica sedimentar apresenta relevância ambiental, pois esta análise proporciona o discernimento entre as possíveis fontes (i.e., terrestre, aquática e antrópica) de MO.

Com base nisso, e com a necessidade de avaliar a qualidade ambiental da porção noroeste da Baía de Vitória (ES), o presente trabalho visa avaliar as fontes naturais e antrópicas da $\mathrm{MO}$ nos sedimentos estuarinos. O sistema estuarino, bem como as bacias hidrográficas tributárias que compõem o gradiente fluvio-estuarino, pode ser considerado um sistema integrado, sendo sua gestão sustentável estratégica para o desenvolvimento socioeconômico da região. 
Lehrback, B. D., et al. (2016). Caracterização através de biomarcadores lipídicos.

\section{MATERIAIS E MÉTODOS}

\section{Área de Estudo}

O sistema estuarino da Baía de Vitória está localizado na Grande Vitória, Espírito Santo $\left(20^{\circ} 14^{\prime}\right.$

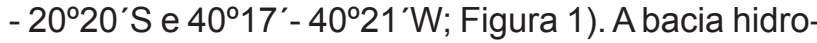
gráfica desse sistema é composta por dez sub-bacias que totalizam uma área de $1.920 \mathrm{~km}^{2}$, com destaque para a bacia do rio Santa Maria da Vitória - SMV (1.536 $\mathrm{km}^{2}$ ) cuja vazão média anual é $14,6 \mathrm{~m}^{3} / \mathrm{s}$. Além da influência fluvial há comunicação com o mar através de dois canais, a Baía de Vitória propriamente dita e o Canal da Passagem, sendo que a hidrodinâmica do sistema estuarino é caracterizada pela ação de micromarés (Saldanha, 2007).

A porção noroeste da baía de Vitoria apresenta manguezais com extensão de $25,6 \mathrm{~km}^{2}$ de área, distribuindo-se ao leste pelo município de Vitória, ao norte pelo município da Serra e ao oeste pelo município de Cariacica. A porção mais ao norte do estuário, próximo aos rios SMV e Bubú, são os locais que apresentam as áreas de manguezais mais preservadas, observandose a presença das espécies Rhizophora mangle (predominante), Laguncularia racemosa e Avicennia germinans (Nalesso et al., 2005; Lima, 2011). As atividades antrópicas desenvolvidas no entorno da região, como: pesca, estrativismo de moluscos e crustáceos, atividades portuárias, turismo, diluição de despejos industriais e de esgotos domésticos; contribuem fortemente com o aumento de poluição orgânica, inorgânica e biológica, sendo que a elevada descarga de esgoto provoca a diminuição do teor de oxigênio dissolvido, e enriquecimento de matéria orgânica no sedimento, o que ocasiona diminuição da diversidade biológica, como nas comunidades faunísticas macrobênticas (Nalesso et al., 2005).

\section{Amostragem}

As estações de amostragem foram definidas através do Projeto "Avaliação Ambiental Integrada de Ambientes Estuarinos: Estudo de Caso do Sistema Estuarino da Grande Vitoria (Espírito Santo) ", através do sistema de informação geográfica ArcGIS 9.3 ESRI. As coletas foram realizadas em 35 estações (Figura 1), sendo essas definidas através de uma grade amostral com equidistância de $500 \mathrm{~m}$, onde nas intercessões desta malha foram definidos os pontos.

A coleta do sedimento superficial (com $5 \mathrm{~cm}$ de profundidade a partir do topo da camada) foi realizada através de um busca fundo do tipo Ekman, e transferindo-as para frascos de vidro previamente calcinados $\left(450{ }^{\circ} \mathrm{C}\right.$ por $2 \mathrm{~h}$ ), com o uso de uma espátula de alumínio. Posteriormente as amostras (acondicionadas em caixas térmicas) foram transportados até laboratório, e congeladas à $-20{ }^{\circ} \mathrm{C}$. A amostragem aconteceu nos dias 16 de setembro (estações 16 a 35) e 28 de outubro (estações de 1 a 15) de 2009.
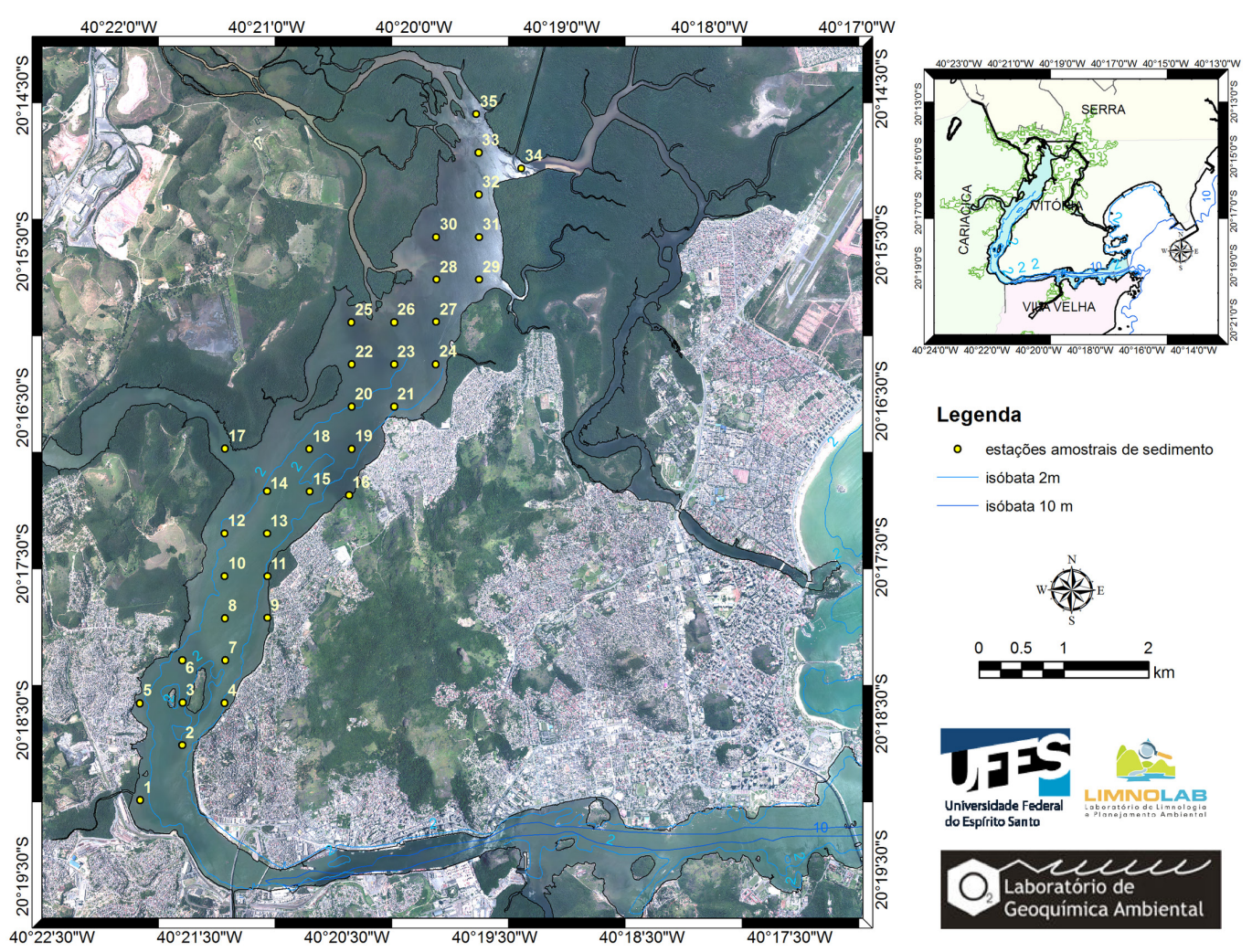

- isóbata $10 \mathrm{~m}$
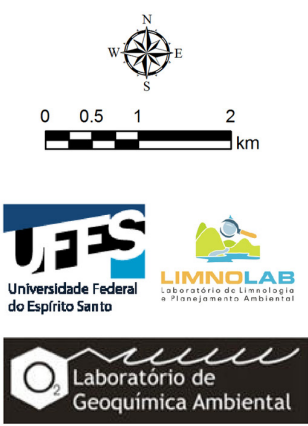

Figura 1 - Estações de coleta amostradas na porção noroeste da Baía de Vitória. 


\section{Determinação de Marcadores Orgânicos}

Foram retiradas alíquotas de aproximadamente $10 \mathrm{~g}$ (peso seco) de cada amostra, previamente liofilizadas e maceradas com grau pistilo de porcelana, sendo os lipídeos extraídos através de uma mistura de solventes destelidos de diclorometano e metanol (9:1, $\mathrm{v} / \mathrm{v}$ ), ambos com grau de pureza PA (para análise), por refluxo em um extrator do tipo Soxhlet durante 24 h. Os extratos resultantes foram pré-concentrados $(\sim 1 \mathrm{~mL})$ através de um evaporador rotativo e, em seguida, secos sob fluxo de nitrogênio gasoso $\left(\mathrm{N}_{2}\right)$. Enxofre foi removido das amostras através de $\mathrm{Cu}$ ativado com $\mathrm{HCl}(\mathrm{PA}) 37 \%$, em seguida, limpo com água bidestilada, metanol e diclorometano, sendo adicionado ao sedimento no momento da extração (Grilo et al., 2013). Os extratos foram submetidos à remoção de água através de uma coluna de $\mathrm{Na}_{2} \mathrm{SO}_{4}$. Por fim, os extratos resultantes foram submetidos à reação de derivação, onde os compostos hidroxilados foram convertidos para trimetilsilil derivativos pela reação com bistrimetilsilil-trifluoracetamida (BSTFA) à uma temperatura de $60{ }^{\circ} \mathrm{C}$ por $40 \mathrm{~min}$. Após esse procedimento todas as amostras, previamente secas novamente por fluxo de $\mathrm{N}_{2}$, foram esterificadas através da adição de $2 \mathrm{ml}$ de uma mistura de metanol e clo- reto de acetila $(9: 1, v / v)$, ambos $P A$, à $40{ }^{\circ} \mathrm{C}$ por um período de $12 \mathrm{~h}$. Em seguida, foram, finalmente, secas sob fluxo de $\mathrm{N}_{2}$ e diluídas em diclorometano até $1 \mathrm{ml}$ antes da injeção no CG-EM.

\section{Análise Instrumental}

Uma alíquota de $1 \mu \mathrm{L}$ de cada extrato bruto foi analisado usando-se um Cromatógrafo a Gás acoplado à Espectrômetro de Massa (CG-EM). A análise em CG-EM foi conduzida através de um CG modelo 7890 acoplado ao EM quadrupolo modelo 5975, Agilent Technologies, equipado com coluna capilar DB-5 com dimensões de $60 \mathrm{~m} \times 250 \mu \mathrm{m} \times 0,25 \mu \mathrm{m}$, da referida empresa. O CG-EM foi operado no modo de ionização dos elétrons (70 eV) e full scan (m/z 50600 ), conforme pode ser visualizado no cromatograma da Figura 2. O método de corrida apresentou como características: temperatura inicial de $60{ }^{\circ} \mathrm{C}$ durante $1 \mathrm{~min}$; primeira rampa de aquecimento a uma taxa de $6{ }^{\circ} \mathrm{C} \cdot \mathrm{min}^{-1}$ até $180^{\circ} \mathrm{C}$, sendo o tempo de elevação de $21 \mathrm{~min}$; a segunda rampa de aquecimento a uma taxa de $2,5^{\circ} \mathrm{C} \cdot \mathrm{min}^{-1}$ até $315^{\circ} \mathrm{C}$, mantendo esta temperatura por $15 \mathrm{~min}$. O tempo total de corrida foi de $90 \mathrm{~min}$. O gás carreador foi o hélio $(\mathrm{He})$, sendo as amostras injetadas no modo splitless (injetor split/splitless).

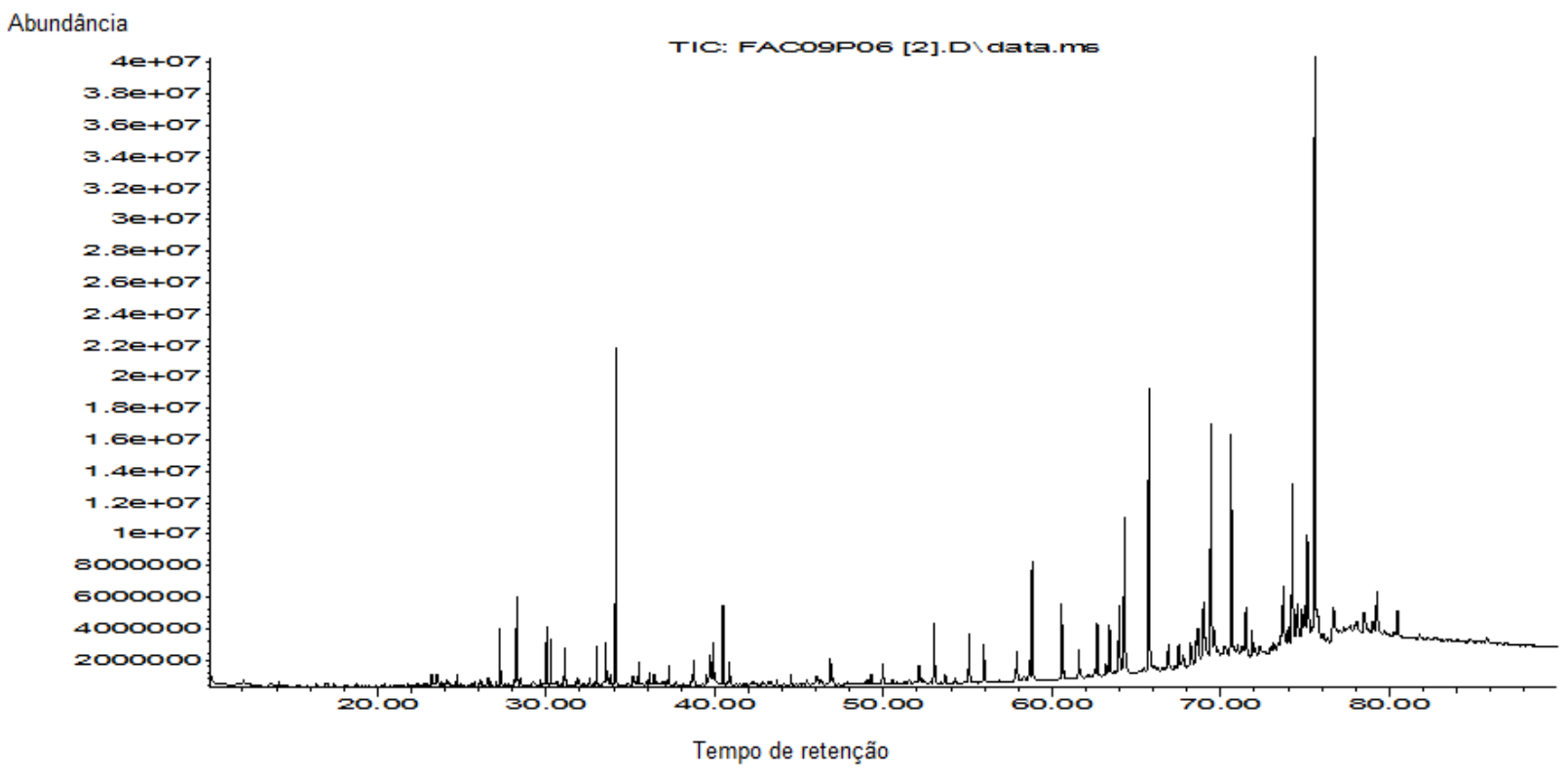

Figura 2 - Cromatograma adquirido no modo full scan do extrato total da amostra FAC09P06.

Para a identificação dos compostos individuais foi utilizado o software MSD ChemStation Data Analysis, sendo realizada através da interpretação da massa espectral (íons base: $\mathrm{m} / \mathrm{z} 129,215$ e 255, para os esteróis; m/z 204 para o taraxerol; m/z 189 para o germanicol; m/z 218, para amerinas; $\mathrm{m} / \mathrm{z} 74$, para ácidos graxos; m/z 57, 71 e 85, para n-alcanos; e m/z 191 para hopanóides), da comparação do espectro de massa da literatura das referências bibliográficas citadas e dos espectros encontrados na biblioteca do equipamento. Apesar de ser identificada a presença de Mistura Complexa Não Resolvida (UCM - Unresolved Complex Mixture), a mesma não foi quantificada através do CG-EM, trata-se apenas de um dado semi-quantitativo.

Para a quantificação dos compostos foi utilizado o padrão interno a-colestano, na concentração de 101 $\mu \mathrm{g} / \mathrm{mL}$, adicionado $100 \mu \mathrm{L}$ às alíquotas analisadas, e 
posteriormente diluídas em $1 \mathrm{~mL}$, resultando em uma concentração final do padrão de 10 ppm para cada amostra. Brancos de laboratório foram utilizados para corrigir possíveis interferentes durante as análises.

Os resultados encontrados, bem como todos os compostos identificados, estão listados na Tabela 1. O limite de detecção (LD) foi de 0,042 ppm, enquanto o limite de quantificação (LQ) foi de 0,139 ppm, sendo obtidos através da razão sinal/ruído e na concentração do padrão a-colestano.

\section{Análise Elementar (razão elementar $\mathrm{C} / \mathrm{N}$ )}

Carbono orgânico total (COT) e nitrogênio total (NT) foram determinados através de um analisador elementar marca Perkin Elmer serie II. As alíquotas (cerca de $1 \mathrm{~g}$ ) foram previamente descarbonatadas através de tratamento com ácido $(\mathrm{HCl} 1 \mathrm{M}$ até $\mathrm{pH}$ $<2,0)$. O sedimento livre de carbonato foi pesado em cápsulas de estanho $(5-10 \mathrm{mg})$ e seguiu para detecção. As análises foram realizadas a partir da combustão das amostras $\left(924^{\circ} \mathrm{C}\right)$, sendo o produto transportado por hélio numa coluna de redução $\left(639{ }^{\circ} \mathrm{C}\right.$ ) onde foram reduzidos em $\mathrm{CO}_{2}, \mathrm{~N}_{2}$ e $\mathrm{H}_{2} \mathrm{O}$. Uma vez reduzidos, os gases foram homogeinizados na zona de mistura no qual, após a despressurização em uma coluna de separação, foram determinados com um detector de condutividade térmica ( $82 \pm 0,05$ ${ }^{\circ} \mathrm{C}$ ). Acetanilida foi usada como padrão de calibração e a precisão média foi de $\pm 0,1 \mathrm{mgC} . \mathrm{g}^{-1}$ para COT e \pm $0,06 \mathrm{mgN} \cdot \mathrm{g}^{-1}$ para NT.

\section{Granulometria}

Uma alíquota de sedimento amostrado foi retirada para a análise da granulometria. Primeiramente, estas subamostras foram peneiradas através de uma peneira de aço inox de malha $2 \mathrm{~mm}$. Em seguida, foram lavadas com água bidestilada para remoção do sal, e deixadas decantar por um período mínimo de 24 h. Após o preparo das amostras, a análise da granulometria foi realizada pelo equipamento Mastersizer 2000, da marca Malvern, que tem como princípio a leitura à lazer. As amostras foram submetidas ao ultrassom (1 min e $30 \mathrm{~s}$ ), para desagregamento das partículas. O equipamento foi configurado para uma rotação de 2500 RPM (rotações por minuto), e a leitura dos resultados foi realizada pelo acessório Hydro 2000 MU.

\section{Análise Estatística Multivariada}

Para melhorar a avaliação dos resultados foi realizada uma análise de correlações entre os índices calculados, dados de concentrações totais das classes lipídicas e concentrações individuais dos compostos, além dos dados de granulometria. O método utilizado para tal correlação foi o teste de Spearman`s Rho $(\rho)$, que analisa as correlações significativas $(P<0,05)$ positivas $(0>\rho>1)$ e negativas $(-1>\rho>0)$ entre as variáveis. As análises foram realizadas através do programa estatístico JMP 8.1.

\section{Análise Espacial}

A interpolação dos dados foi realizada através do sistema de informações geográfica ArcGIS 9.3, ESRI, através do método Spline. O método se baseia na interpolação através de uma função matemática de base radial, que minimiza a curvatura superficial, através de uma combinação linear ponderada dos valores das amostras (Johnston et al., 2001), resultando em uma superfície mais suave, o chamado efeito smooth (Isaaks \& Srivastava, 1989).

Modelo de superfície contínua foi gerado a partir dos dados para o teor de lama. A área resultante para interpolação dos dados foi de $9,2 \mathrm{~km}^{2}$, e os critérios para interpolação pelo Spline foram peso 0,1 e número de pontos vizinhos interpolados 12 .

\section{RESULTADOS E DISCUSSÕES}

A composição de lipídeos no sedimento de fundo depende da espécie biológica fonte e das influências químicas, físicas e microbiológicas, que afetam ou modificam tais compostos durante, e após, a deposição (Mackenzie et al., 1982). Elevado aporte de detritos de plantas superiores terrestres é caracterizado pela presença de lipídeos alifáticos de cadeia longa (séries homólogas de n-alcanos, ácidos graxos e álcoois), variando entre $\mathrm{C}_{20}-\mathrm{C}_{35}$, e principalmente pela presença de pentacíclicos triterpenóides (Abu Bakar et al., 2011).

A distribuição de ácidos graxos (AG) que caracteriza contribuição de plantas superiores (ou seja, material terrestre) ocorre pela presença de compostos de cadeias longas $\left(>C_{22}\right)$, com predominância de cadeias pares em relação às ímpares, conforme apresentado na Figura 3 (Meziane \& Tsuchiya, 2000; Kristensen et al., 2008). Dentre os compostos de cadeias longas se destacam o $\mathrm{C}_{24: 0}, \mathrm{C}_{26: 0}$ e $\mathrm{C}_{28: 0}$, que além de observados em outros tipos de plantas superiores, são os ácidos mais abundantes nas ceras epicuticulares das folhas de mangue (Bourbonniere \& Meyers, 1996; Kristensen et al., 2008).

Também foi observada a presença de ácidos saturados de cadeias curtas (SCFA) variando entre $\mathrm{C}_{10: 0}$ e $\mathrm{C}_{21: 0}$. Os compostos que se destacaram entre os de cadeia curta são o $C_{14: 0}, C_{16: 0}$ e $C_{18: 0}$. Sendo o $\mathrm{C}_{16: 0}$ (ácido palmítico) o composto dominante dentre os AG, apresentando concentração que variou entre

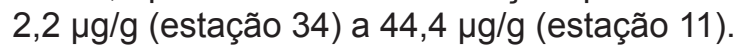



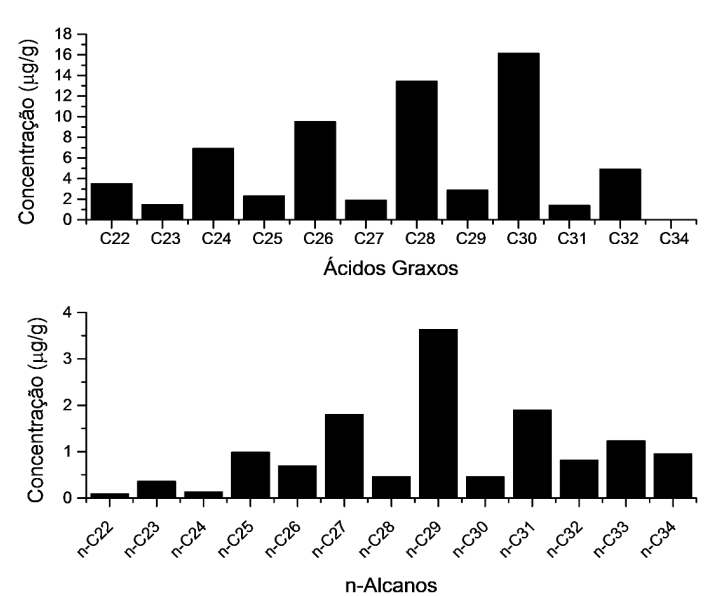

Figura 3: Distribuição de ácidos graxos e n-alcanos que representa aporte terrestre.

Ácidos graxos mono-insaturados (MonoUnsaturated Fatty Acids - MUFA) - $C_{16: 1}, C_{17: 1}$ e $C_{18: 1}$ - também foram observados na área estudada, sendo o $C_{18: 1 \omega 9}$ e $C_{18: 1 \omega 7}$ os mais abundantes dentre os MUFAs, com concentrações máximas em $7,7 \mu \mathrm{g} / \mathrm{g}$ e $8,1 \mu \mathrm{g} / \mathrm{g}$, ambos na estação 13. Esta se encontra próxima à desembocadura do rio Bubu (Figura 1), que contribui para o aporte de nutrientes local, fazendo com que haja uma maior produtividade primária, aumentando a concentração destes e de outros marcadores aquáticos (planctônicos), como os ácidos $C_{14: 0}$ e $C_{18: 0}$, citados anteriormente. Estes MUFAs são compostos característicos de origem planctônica (Canuel, 2001). O ácido $\mathrm{C}_{16: 1 \omega 7}$ é o composto predominante em diatomáceas (Volkman, 1986).

Já os compostos ramificados $C_{15}$ e $C_{17}$ iso e anteiso, representam AG característicos de bactérias, sendo abundantes naquelas redutoras de sulfato (Volkman et al., 1998; Rontani \& Volkman, 2005). Estes compostos foram observados próximos a margem da região urbanizada e do deságue de esgotos domésticos (Jesus et al., 2004), que pode provocar a formação de um ambiente subóxico ou anóxico, favorecendo o desenvolvimento de bactérias, que também se caracteriza por ser uma região de baixa acumulação de material (estação 34).

$A$ razão entre compostos terrestres e aquáticos (RTA), calculada para os AG $\left(\right.$ RTA $_{\text {ác.graxos }}=$ $\left.\left(C_{24}+C_{26}+C_{28}\right) /\left(C_{12}+C_{14}+C_{16}\right)\right)$, é um índice comumente utilizado na avaliação das fontes de matéria orgânica para o ambiente. Baixos valores $(<1)$ indicam contribuição preferencial do aporte aquático, enquanto que valores maiores do que a unidade indica aporte terrestre (Bourbonniere \& Meyers, 1996).

Os valores encontrados para a RTA ác.graxos variaram entre 0,2 (estação 35) e 2,6 (estação 9). Algumas estações apresentaram valores de RTA que caracterizam aporte autóctone. No entanto, estes devem ser considerados com cautela, pois o ácido $C_{16: 0}$ (ácido palmítico) também é observado em folhas de mangue (Volkman et al., 1998; Meziane \& Tsuchiya, 2000; Kristensen et al., 2008) e outros organismos, como algumas bactérias (Volkman et al., 1998). Esses valores mais baixos de RTA foram observados com mais frequência nas estações na porção mais ao sul da Baía, onde o aporte de nutrientes é elevado em função da proximidade com as áreas urbanas e deságue de esgotos domésticos, possibilitando um aumento da concentração fitoplanctônica. Jesus et al. (2004) também associou a distribuição de metais nesta região ao aporte de esgoto.

Os dados para Índice Preferencial de Cabono (IPC ác.graxos $=\left\{\left[\left(\sum_{\text {pares }} \mathrm{C}_{12}-\mathrm{C}_{20}\right) /\left(\sum_{\text {impares }} \mathrm{C}_{13}\right.\right.\right.$ $\left.\left.\left.\left.\mathrm{C}_{21}\right)\right]+\left[\left(\sum_{\text {pares }} \mathrm{C}_{12}-\mathrm{C}_{20}\right) /\left(\sum_{\text {impares }} \mathrm{C}_{13}-\mathrm{C}_{19}\right)\right]\right\} / 2\right)$ representam uma ferramenta complementar na caracterização das fontes de material para o ambiente. O mesmo variou entre 1,2 (estação 27) e 18,4 (estação 16). Assim como o IPC $\left.\left.\left.\left.\mathrm{C}_{24}-\mathrm{C}_{32}\right)\right]+\left[\left(\sum_{\text {impares }} \mathrm{C}_{23}-\mathrm{C}_{33}\right) /\left(\sum_{\text {pares }} \mathrm{C}_{22}-\mathrm{C}_{32}\right)\right]\right\} / 2\right)$ que variou entre 1,3 (estação 12) até 7,6 (estação 21), em maioria apresentando valores maiores que a unidade, sendo que os valores elevados (entre 5 e 10), indicam contribuição de plantas vasculares (Jeng, 2006). Entretanto, outro índice calculado para n-alcanos, o ACL (Average chain-lenght) $\left(\mathrm{ACL}=\left[\left\{25\left(\mathrm{C}_{25}\right)+27\left(\mathrm{C}_{27}\right.\right.\right.\right.$ )$\left.\left.\left.+29\left(C_{29}\right)+31\left(C_{31}\right)+33\left(C_{33}\right)\right] /\left(C_{25}+C_{27}+C_{29}+C_{31}+C_{33}\right)\right\}\right)$, apresentou valores variando de 28,13 (estação 17) até 29,93 (estação 10), sendo que estes podem indicar tanto contribuição de aporte petrogênico (valores $A C L ~ ~ 28)$, quanto influência de vegetais superiores (valores entre 29 e 30) (Jeng, 2006). O referido autor observou os primeiros resultados em amostras marinhas, e os últimos em sedimentos fluviais.

Distribuição de n-alcanos também pode ser utilizada para caracterizar o tipo de fonte aportada para ambientes estuarinos. Aquela que evidencia aporte terrestre é marcada pela presença de compostos de cadeias longas $\left(\mathrm{C}_{22}\right.$ até $\left.\mathrm{C}_{34}\right)$, com predominância de compostos de cadeia ímpar e máximos nos compostos $\mathrm{C}_{29}$ e $\mathrm{C}_{31}$, embora algumas amostras também possam apresentar máximos em $\mathrm{C}_{27}$. Enquanto que, o tipo de distribuição que caracteriza aporte marinho é marcado pela presença de compostos de cadeias curtas $\left(\mathrm{C}_{12}-\mathrm{C}_{21}\right)$. Já o tipo de distribuição que apresenta a presença de compostos variando entre $\mathrm{C}_{10}-\mathrm{C}_{34}$, onde não há predominância de cadeias pares ou ímpares, indica aporte por fontes petrogênicas (Jeng, 2006). No presente estudo foi observado um tipo de distribuição típico de influência terrestre, conforme pode ser observado no gráfico da Figura 3.

Sabe-se que a razão elementar entre carbono orgânico total e nitrogênio elementar $(\mathrm{C} / \mathrm{N})$ é uma 
importante ferramenta auxiliar na identificação da predominância entre as fontes, sendo que os valores de $\mathrm{C} / \mathrm{N}$ entre 4 e 10 indicam origem planctônica (fito e zoo), enquanto que valores maiores que 20 indicam aporte terrestre proveniente de plantas superiores (Meyers, 1994). A partir destes resultados pode-se perceber que a maioria das estações (Figura 4) apresentaram valores intermediários, entre 10 e 20 , sendo que os mesmos podem indicar um ambiente de transição, apresentando múltiplas fontes (Mahapatra et al., 2011), o que está de acordo com as características de um estuário. Esta característica evidencia aporte de material proveniente tanto de planta superior como de material produzido no estuário (autóctone).

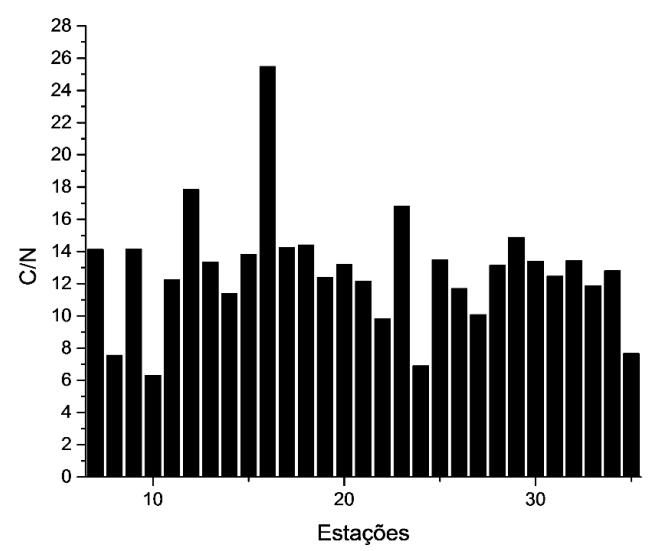

Figura 4 - Valores de C/N para as estações de amostragem.

A partir dos dados expostos pode-se perceber que as estações que apresentaram valores indicando origem planctônica ( $\mathrm{C} / \mathrm{N}$ entre 4 e 10), se localizam na porção ao sul da área de estudo (pontos 8 e 10), que se caracteriza pelo aporte de esgotos domésticos; bem como na região ao norte (pontos 24 e 35), que ocorrem próximo a desembocadura fluviais, ou seja, recebem input de nutrientes favorecendo o desenvolvimento fitoplanctônico. Já a região que apresenta valor característico de aporte terrestre é observada na estação 16, que se localiza próxima a margem, local de maior deposição de material.

Outra importante característica na avaliação da $\mathrm{MO}$, e que caracteriza fontes de origem terrestre, é através do uso de pentacíclicos triterpenóides (PT). Estes compostos são encontrados em abundância em folhas de mangue e outros tipos de vegetação superior (Dodd et al., 1995; Dodd et al., 1998; Koch et al., 2003; Kristensen et al., 2008). A relativa estabilidade destes compostos durante a sedimentação e diagênese viabiliza sua utilização (Simoneit, 1986).

Dentre os triterpenóides indicadores de plantas superiores identificados, o composto dominante foi a $\beta$-amirina (olean-12-an-3 $\beta$-ol), e teve sua concen- tração variando entre 4,0 $\mu \mathrm{g} / \mathrm{g}$ (estação 10) e 87,2 $\mu \mathrm{g} / \mathrm{g}$ (estação 21). Outros PT identificados foram: $\alpha$-amirina (urs-12-an-3 $\beta$-ol), taraxerol (taraxer-14-

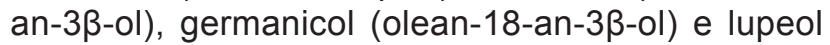
(lup-20(29)-an-3ß-ol).

O taraxerol e a $\beta$-amirina apresentaram uma boa correlação de Spearman's Rho $(\rho)$ positiva ( $\rho$ $=0,8849 ; \mathrm{P}<0,05$ ) sugerindo que ambos possuem a mesma origem ou sofrem os mesmos processos de degradação e de deposição. Apesar da elevada resistência à degradação microbiológica do taraxerol, quando comparados com compostos do tipo oleanona (Killops \& Frewin, 1994), este composto foi encontrando em menor concentração em relação a $\beta$-amirina em todas as estações amostradas. Isto pode ter ocorrido em função de uma rápida transformação diagenética do taraxerol para $\beta$-amirina (Killops \& Frewin, 1994). Este processo de conversão pode ocorrer no sedimento através da isomerização catalisada por ácido (Ten Haven \& Rullkötter, 1988). Outros autores também observaram a predominância da $\beta$-amirina ao longo de uma sequência sedimentar (Ten Haven \& Rullkötter, 1988; Ten Haven et al., 1992a; Ten Haven et al., 1992b; Killops \& Frewin, 1994).

Alguns tipos de esteróis também são utilizados para avaliar a contribuição da vegetação terrestre para o ambiente (Koch et al., 2003). Dentre os esteróis aqueles que melhor caracterizam este aporte são: estigmasterol (24-etilcolesta-5,22E-dian-3 $\beta$-ol $-\mathrm{C}_{29} \Delta^{5,22}$ ), campesterol (24-metilcolest-5-an-3 $\beta$-ol $\mathrm{C}_{28} \Delta^{5}$ ) e o $\beta$-sitosterol (24-etilcolest-5-an-3ß-ol $-\mathrm{C}_{29} \Delta^{5}$ ) (Volkman, 1986; Ten Haven et al., 1992b), onde suas concentrações podem ser observadas na Tabela 1. Todos foram identificados na área de estudo, sendo que o $\beta$-sitosterol apresentou correlação positiva com o campesterol $(\rho=0,5241 ; P<0,05)$, bem como estigmasterol apresentou com campesterol $(\rho=0,5872$; $P$ $<0,05$ ), o que também sugere que estes compostos possivelmente originaram da mesma fonte ou sofreram os mesmos processos de degradação e deposição. Embora estes também possam ser encontrados em alguns organismos planctônicos, sua predominância está relacionada com plantas superiores (Volkman, 1986).

O colesterol (colest-5-an-3 $\beta$-ol $-\mathrm{C}_{27} \Delta^{5}$ ) foi 0 esterol dominante dentre os indicadores de fontes aquáticas, distribuindo-se entre a maioria das estações amostradas. A concentração deste composto variou entre $0,1 \mu \mathrm{g} / \mathrm{g}$ (estação 34 ) até $4,7 \mu \mathrm{g} / \mathrm{g}$ (estação 2). Este tipo de composto indica origem animal, sendo associado à produção por zooplâncton (McCallister et al., 2006), principalmente crustáceos (Carreira et al., 2009), embora também existem evidências de sua presença em algumas microalgas (Volkman, 1986). Além da origem natural o colesterol também pode ser 
Braz. J. Aquat. Sci. Technol., 2016, 20(1).

Tabela 1 - Concentração $\mu \mathrm{g} / \mathrm{g}$ dos compostos identificados.

\begin{tabular}{|c|c|c|c|c|c|c|c|c|c|c|}
\hline 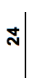 & 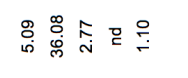 & 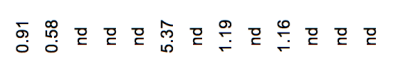 & g & ㄹ. & ㅁ & g. & : & 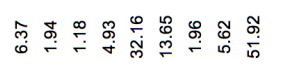 & 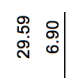 & \\
\hline$\approx$ & 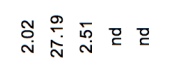 & 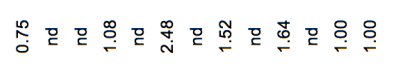 & g & $\begin{array}{l}\hat{i} \\
\text { of }\end{array}$ & ㅁ & $\stackrel{\sim}{\stackrel{\rho}{\sim}}$ & 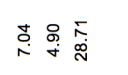 & 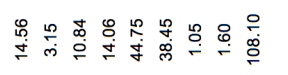 & 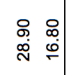 & \\
\hline$\approx$ & 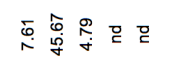 & 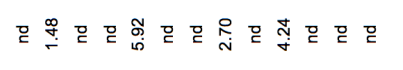 & $\stackrel{\infty}{\infty}$ & 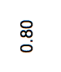 & 모 & 몯 & 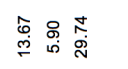 & 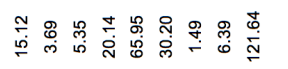 & \begin{tabular}{lll}
0 & $\infty$ \\
$\stackrel{\rho}{c}$ & $\infty$ \\
\hdashline & $\infty$
\end{tabular} & \\
\hline $\bar{\sim}$ & 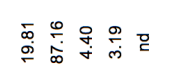 & 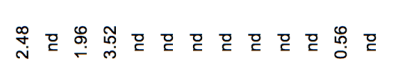 & ? & 맏 & $\stackrel{\hat{N}}{i}$ & 모 & 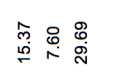 & 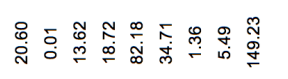 & 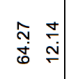 & \\
\hline ๙ & 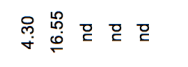 & 믇 묻 & 무 & হ & T) & ㄹ & $\bar{q}$ & 品 㐘 & $\begin{array}{ll}7 \\
\dot{5}\end{array}$ & \\
\hline ๑ & 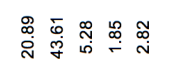 & 묻 윧 & 总 & 蛋 & 모 & 룯 & 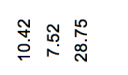 & 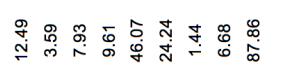 & 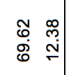 & \\
\hline 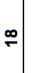 & 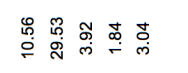 & 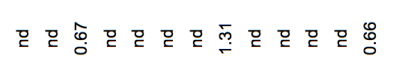 & 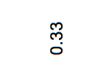 & 号 & 몸 & 모 & 曽 & 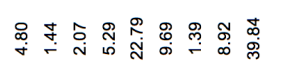 & 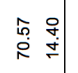 & \\
\hline$\approx$ & 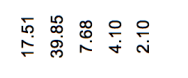 & 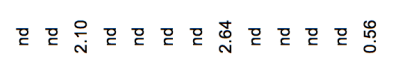 & $\stackrel{8}{\circ}$ & 무 & 모 & 무 & 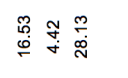 & 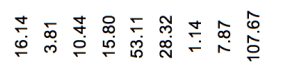 & 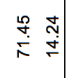 & \\
\hline$\stackrel{0}{\circ}$ & 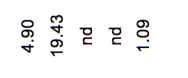 & 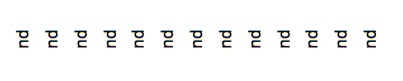 & চ্口: & $\stackrel{\text { g }}{\circ}$ & กู่ & 总 & $\underset{j}{\stackrel{D}{\sim}} \stackrel{\sim}{\sim} \underset{\sim}{\infty}$ & 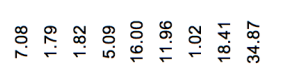 & 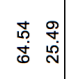 & \\
\hline$\stackrel{2}{\circ}$ & 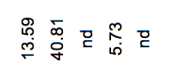 & 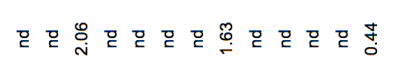 & $\stackrel{\leftrightarrow}{\circ}$ & $\stackrel{i}{i}$ & ㅁ & c & 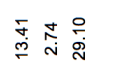 & 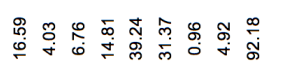 & $\begin{array}{ll}\stackrel{\infty}{\infty} \\
\stackrel{\infty}{\sim} & \stackrel{\infty}{\sim}\end{array}$ & \\
\hline 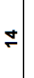 & 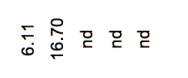 & 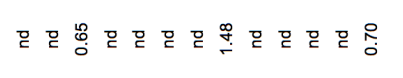 & 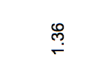 & $\stackrel{\ulcorner}{\check{c}}$ & is & $\tilde{\Xi}$ & 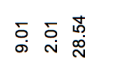 & 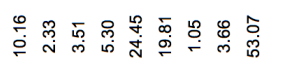 & 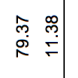 & \\
\hline$\stackrel{m}{=}$ & 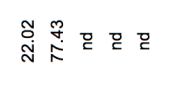 & 므 몯 & $\stackrel{\infty}{N}$ & $\stackrel{\Xi}{\leftarrow}$ & 모 & 무 & 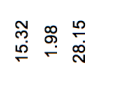 & 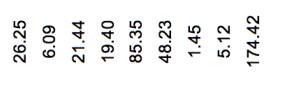 & 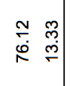 & \\
\hline$\simeq$ & 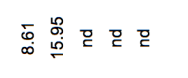 & 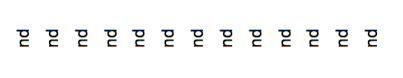 & $\stackrel{\leftrightarrow}{\stackrel{\leftrightarrow}{\circ}}$ & $\stackrel{\infty}{\leftarrow}$ & $\stackrel{0}{0}$ & $\begin{array}{c}\infty \\
\infty \\
0\end{array}$ & 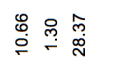 & 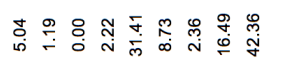 & 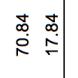 & \\
\hline$=$ & 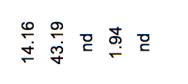 & 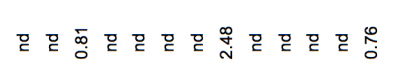 & $\stackrel{\infty}{\stackrel{\infty}{*}}$ & $\stackrel{\circ}{\circ}$ & $\stackrel{\leftrightarrow}{\stackrel{\leftrightarrow}{~}}$ & $\underset{\text { N }}{\text { N }}$ & 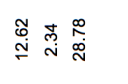 & 妾 & 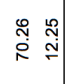 & \\
\hline$\div$ & 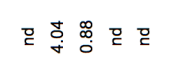 & 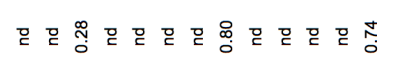 & $\stackrel{ }{ }$ & 모 & 모 & 무 & 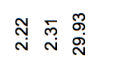 & 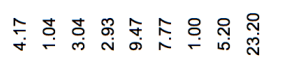 & $\stackrel{8}{:}$ & \\
\hline ๑) & 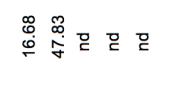 & 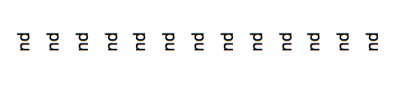 & 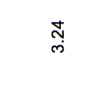 & $\stackrel{\text { S. }}{\mathrm{N}}$ & $\stackrel{\stackrel{m}{\rightleftarrows}}{=}$ & $\stackrel{\hat{o}}{\stackrel{-}{r}}$ & 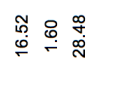 & 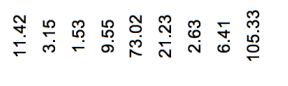 & 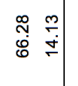 & \\
\hline$\infty$ & 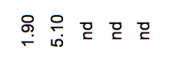 & 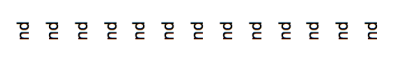 & g্ & 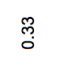 & $\frac{9}{0}$ & ָู & 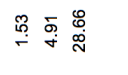 & 启㣽 & 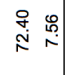 & \\
\hline$\uparrow$ & $\stackrel{\circ}{\circ} \stackrel{\circ}{\circ}$ & 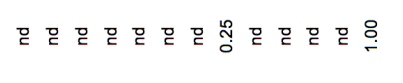 & $\stackrel{R}{2}$ & $\ddot{\circ}$ & స్. & 둘 & 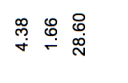 & 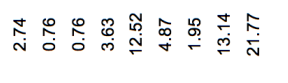 & $\begin{array}{ll}\stackrel{N}{\mathfrak{N}} \\
\stackrel{\infty}{\dot{j}} \\
\stackrel{+}{\leftarrow}\end{array}$ & \\
\hline - & 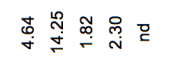 & 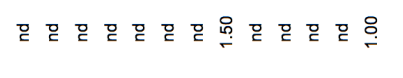 & 무 & 몯 & ? & 무 & 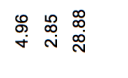 & 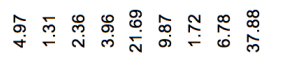 & 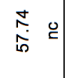 & 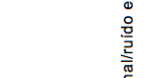 \\
\hline ه & 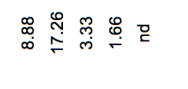 & 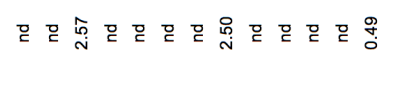 & $\stackrel{\overline{i n}}{\stackrel{i}{r}}$ & $\stackrel{t}{\check{r}}$ & $\underset{\mathrm{N}}{\stackrel{J}{N}}$ & $\stackrel{\infty}{\underset{N}{N}}$ & 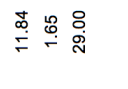 & 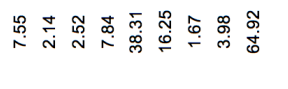 & $\stackrel{\infty}{\infty} \underset{\infty}{\infty}=$ & 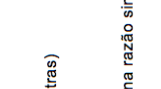 \\
\hline+ & 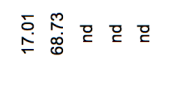 & 무 묻 묻 묻 묻 & $\stackrel{8}{\leftrightarrow}$ & $\stackrel{\stackrel{\leftrightarrow}{\sim}}{\sim}$ & 맘 & 무 & 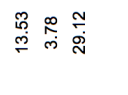 & 息 & $\underset{c}{F} \stackrel{0}{c}$ & 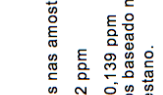 \\
\hline m & 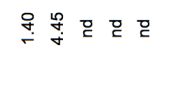 & 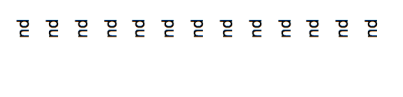 & ๙̊ & $\underset{\infty}{\infty}$ & 무 & c & 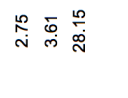 & 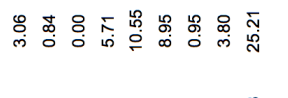 & $\underset{\substack{\infty \\
\stackrel{\infty}{i}}}{\stackrel{0}{c}}$ & 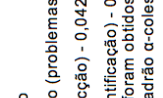 \\
\hline$\sim$ & 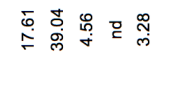 & 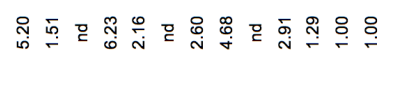 & 로 & $\stackrel{\substack{\infty \\
0}}{0}$ & 巳 & 모 & 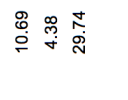 & 矛离 & $\stackrel{\circ}{\stackrel{+}{\perp}} \stackrel{0}{\complement}$ & 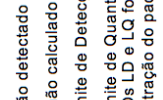 \\
\hline - & 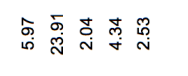 & 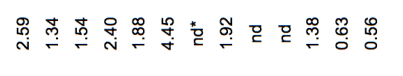 & $\stackrel{\circ}{\circ}$ & $\stackrel{8}{\circ}$ & ㄹ & 핟 & 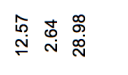 & 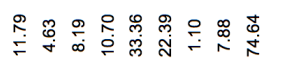 & 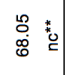 & 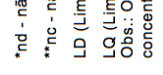 \\
\hline & 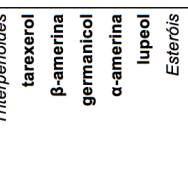 & 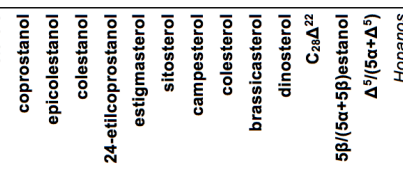 & 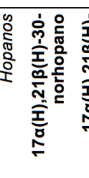 & 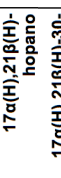 & 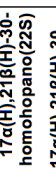 & 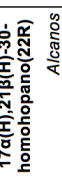 & 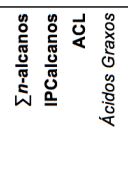 & 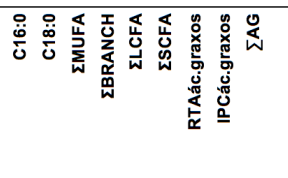 & 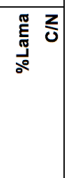 & \\
\hline
\end{tabular}


Continuação:

\begin{tabular}{|c|c|c|c|c|c|c|c|c|c|c|}
\hline$\stackrel{\infty}{\infty}$ & 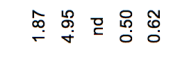 & 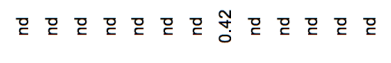 & g & g & 맘 & 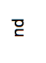 & 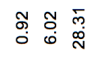 & 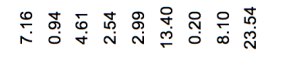 & 芯 : : & \\
\hline & 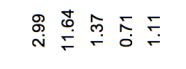 & 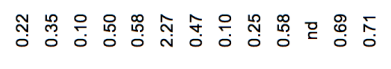 & g & ฐ & 巳 & q & 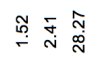 & 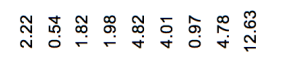 & $\begin{array}{ccc}\widetilde{0} & : \\
\stackrel{0}{\circ} & \stackrel{\sim}{\sim}\end{array}$ & \\
\hline & 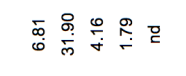 & 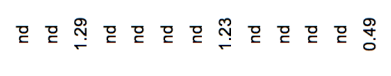 & $\stackrel{\infty}{8}$ & $\stackrel{0}{\circ}$ & 巳 & 巳 & 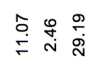 & 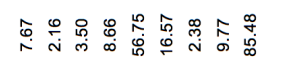 & $\stackrel{\substack{0 \\
\hdashline}}{=}$ & \\
\hline & 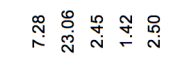 & 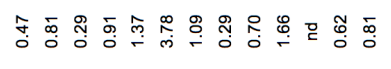 & g & 맏 & g & 巳 & 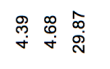 & 芦 & 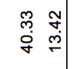 & \\
\hline & 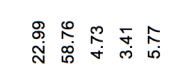 & 몯 & g. & ㅁ. & ․ & 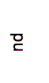 & 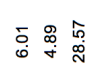 & 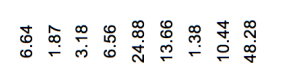 & 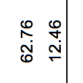 & \\
\hline & 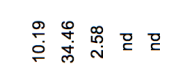 & 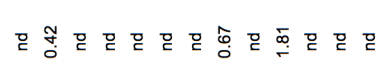 & 巳 & $\nsubseteq$ & g & 巳 & 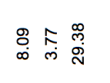 & 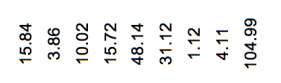 & 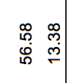 & \\
\hline & 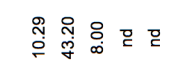 & 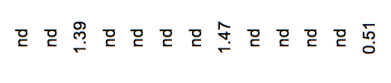 & פ & $\because$ & פ & 巳 & 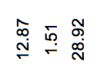 & 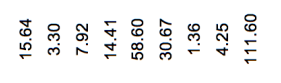 & 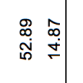 & \\
\hline & 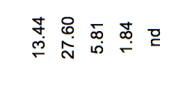 & 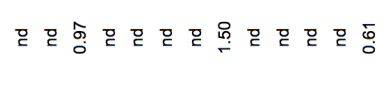 & $\stackrel{\substack{0 \\
\hdashline}}{\circ}$ & $\overline{\bar{g}}$ & 巳 & 巳 & 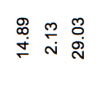 & 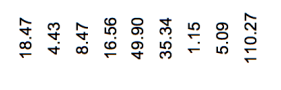 & 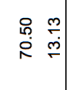 & 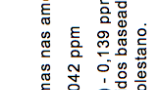 \\
\hline & 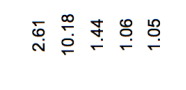 & 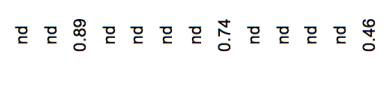 & $\grave{\circ}$ & 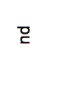 & g & 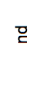 & 营总 & 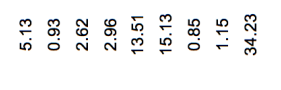 & $\stackrel{\circ}{\stackrel{\circ}{\infty}} \stackrel{0}{\circ} \stackrel{0}{\circ}$ & \\
\hline & 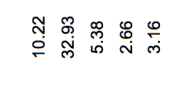 & 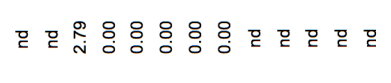 & $\stackrel{\substack{\infty \\
0}}{0}$ & $\stackrel{\infty}{\infty}$ & : & g & 总离 & 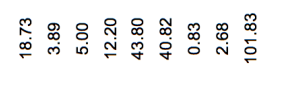 & ث̊: & 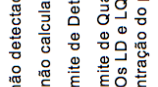 \\
\hline & 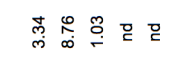 & 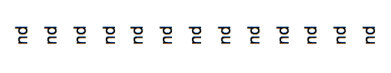 & $\frac{\stackrel{\circ}{0}}{0}$ & $\stackrel{\circ}{0}$ & g & ¿ & 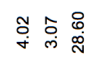 & 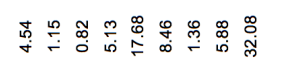 & 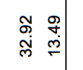 & $\begin{array}{ll} & \\
0\end{array}$ \\
\hline & & 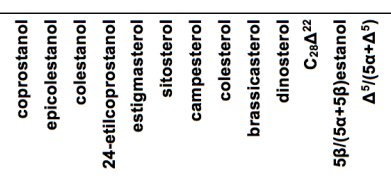 & 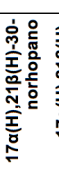 & & & & 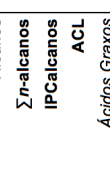 & 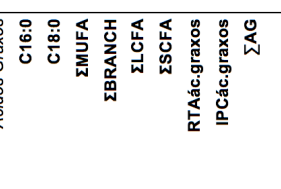 & 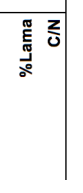 & \\
\hline
\end{tabular}

associado ao aporte por esgotos domésticos (Silva et al., 2008), sendo observado nas fezes de alguns mamíferos, incluindo o homem (Leeming et al., 1996). Sendo que, sua predominância pode ser justificada pela abundância de organismos zooplanctônicos (água doce, estuarinos, costeiros e oceânicos) no sistema estuarino da Baía de Vitória, sendo os copépodas dominantes na região de estudo (Sterza \& Fernandes, 2006).

Além do colesterol $\left(\mathrm{C}_{27} \Delta^{5}\right)$ também foram observados os compostos brassicasterol (24-metilcolesta5,22E-dian-3 $\beta$-ol $\left.-\mathrm{C}_{28} \Delta^{5,22}\right)$, dinosterol $(4 \alpha, 23,24$ -

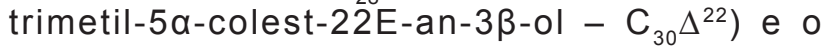
24-metilcoleta-22-an-3 $\beta$-ol $\left(\mathrm{C}_{28} \Delta^{22}\right)$. O brassicasterol $\left(\mathrm{C}_{28} \Delta^{5,22}\right)$ é encontrado no fitoplâncton, e pode ser utilizado como biomarcador para diatomáceas, uma vez que representa mais de $90 \%$ do total de esteróis encontrados neste organismo (Volkman, 1986). O dinosterol é um biomarcador específico para dinoflagelados, embora ele não esteja presente em todas as espécies (Volkman, 2003). Já o $\mathrm{C}_{28} \Delta^{22}$ (24-metilcolet22-an-3ß-ol) pode ser utilizado como biomarcador de fitoplâncton em geral (Jaffé et al., 1995), embora também possa ser biossintetizado por plantas terrestres, além de outros tipos de algas (Xu et al., 2006). Apesar destes compostos não terem apresentado uma correlação entre si, eles podem estar relacionados à mesma fonte, segundo as literaturas citadas.

Sabe-se que estruturas lipídicas são susceptíveis a degradação (Mannino \& Harvey, 1999), assim, a labilidade dos esteróis pode explicar a depleção destes compostos em certas estações. Assim como para os esteróis, a depleção de n-alcanos de cadeia curta (origem planctônica) pode ser explicada pela labilidade destes compostos, já que eles são mais facilmente degradados do que os compostos de cadeia longa (Bourbonniere \& Meyers, 1996). Através da análise granulométrica pode-se perceber que na região de estudo predomina sedimento lamoso (Figura 5), e através da correlação estatística realizada para o somatório de cada classe lipídica (AG, n-alcanos e triterpenóides), constatou-se que as maiores concentrações se relacionam positivamente com menores granulometrias (Tabela 2). Dessa forma, como na estação 35 foi observada uma elevada porcentagem de areia, esta apresentou uma baixa correlação com 
a concentração dos compostos em geral, uma vez que a matéria orgânica adere mais facilmente em partículas finas.

Tabela 2 - Relação entre a granulometria e as concentrações totais (n-alcanos, AG e terpenóides; Correlação de Spearman, $P<0,05$ ).

\begin{tabular}{c|c|c}
\hline $\begin{array}{c}\text { Variável 1 } \\
\text { Granulometria } \\
\text { (fi) }\end{array}$ & $\begin{array}{c}\text { Variável 2 } \\
\text { Conc. Totais }\end{array}$ & $\begin{array}{c}\boldsymbol{R} \boldsymbol{h o} \\
(\boldsymbol{\rho})\end{array}$ \\
\hline 0,7 & $\sum n$-alcanos & 0,3849 \\
0,97 & $\sum$ Terpenóides & 0,3515 \\
0,97 & $\sum n$-alcanos & 0,4339 \\
1,4 & $\sum n$-alcanos & 0,3896 \\
1,95 & $\sum n$-alcanos & 0,3482 \\
22,5 & $\sum n$-alcanos & 0,3927 \\
31,25 & $\sum$ Terpenóides & 0,4238 \\
31,25 & $\sum A G$ & 0,3838 \\
31,25 & $\sum n$-alcanos & 0,5465 \\
45 & $\sum A G$ & 0,5345 \\
45 & $\sum n$-alcanos & 0,6627 \\
45 & $\sum$ Terpenóides & 0,5773 \\
62,5 & $\sum$ Terpenóides & 0,6535 \\
62,5 & $\sum n$-alcanos & 0,7625 \\
62,5 & $\sum A G$ & 0,7104 \\
90 & $\sum n$-alcanos & 0,6176 \\
90 & $\sum A G$ & 0,6765 \\
90 & $\sum$ Terpenóides & 0,4594 \\
125 & $\sum A G$ & 0,4126 \\
125 & $\sum n$-alcanos & 0,3574 \\
\hline &
\end{tabular}

Ambientes aquáticos que recebem efluentes domésticos e industriais tem sua qualidade comprometida, o que acarreta em riscos para a saúde humana e afeta a sobrevivência dos organismos que habitam o local. Dessa forma, a utilização de biomarcadores antrópicos é importante para verificar tal qualidade (Carreira et al., 2004), já que os mesmos se caracterizam pela sua elevada especificidade em relação à fonte e boa resistência à destruição bacteriana (Takada et al., 1997).

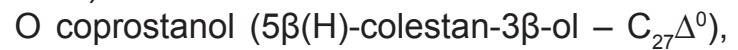
presente na $\mathrm{MO}$ sedimentar, representa poluição oriunda de esgotos domésticos, uma vez que está presente nas fezes de animais superiores, como humanos e outros tipos de mamíferos, como resultado da hidrogenação (redução) do colesterol no intestino destes animais, mediado pela ação bacteriana

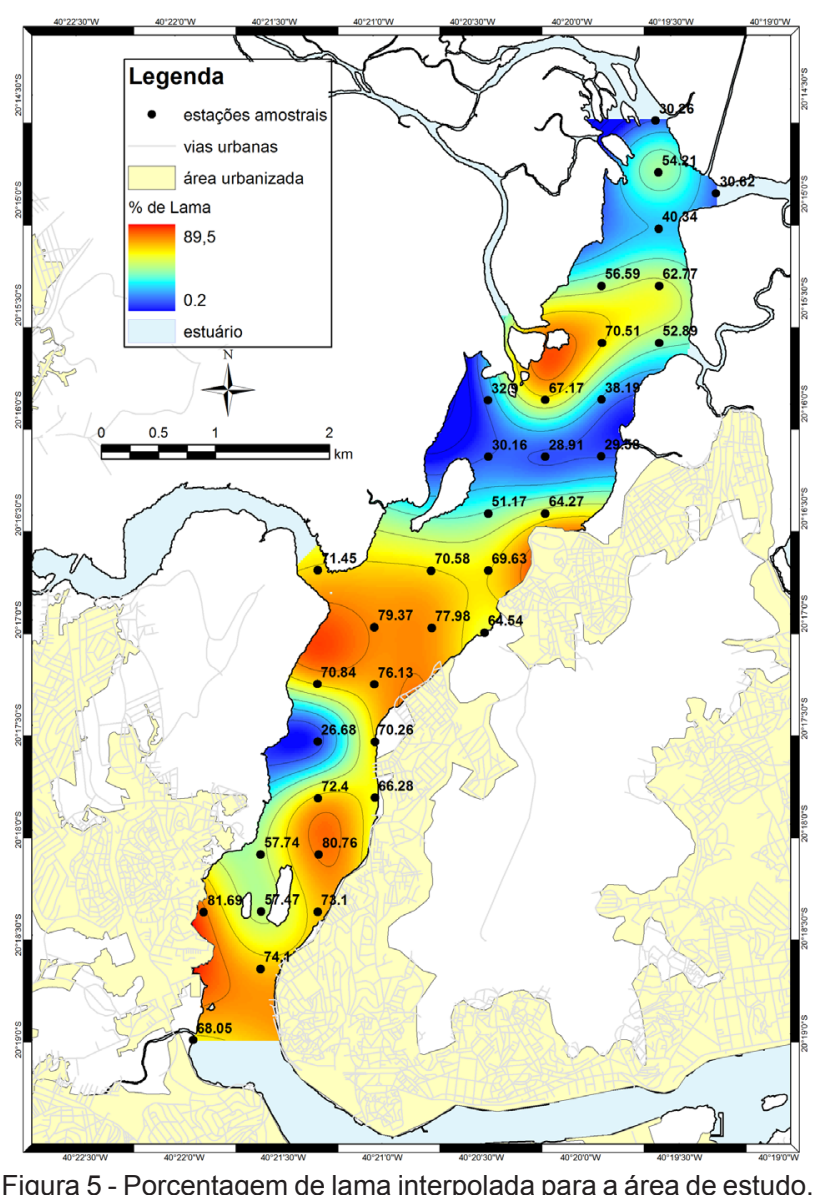

Figura 5 - Porcentagem de lama interpolada para a área de estudo.

(Leeming et al., 1996). Ele representa cerca de $60 \%$ do total de esteróis fecais presentes nas fezes humanas (Leeming et al., 1996).

O colestanol ( $5 \alpha$-colestan-3 $\beta$-ol) e o 24-etilco-

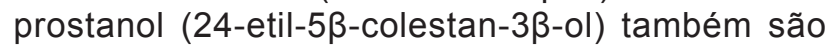
utilizados como biomarcadores fecais, sendo encontrados nas fezes de alguns mamíferos, como porcos, humanos e herbívoros (Leeming et al., 1996). O colestanol, também pode ser derivado através de reações diagenéticas do colesterol, já que a ação microbiológica converte estenois para estanois (Mackenzie et al., 1982), que pode ser determinado através da razão entre estenois/estanois. A referida razão não pôde ser calculada para a maioria das estações deste estudo, sendo o valor observado de 0,8 (estação 1), indicando que nesta região pode estar ocorrendo degradação bacteriana, de acordo com o trabalho realizado por Canuel e Martens (1993), que observaram valores entre $0,1-0,5$ indicando tal processo.

Já o 24-etilcoprostanol é o marcador fecal dominante nas fezes dos herbívoros (Leeming et al., 1996).

No presente trabalho foi observada a presença de fecoesteróis que evidenciam tal aporte antrópico. O coprostanol $\left(\mathrm{C}_{27} \Delta^{0}\right)$, principal indicador de material 
fecal, apresentou sua concentração variando entre $0,2 \mu \mathrm{g} / \mathrm{g}$ (estação 34) - 5,2 $\mu \mathrm{g} / \mathrm{g}$ (estação 2). Já o colestanol $\left(\mathrm{C}_{27}{ }^{0}\right)$ e o epicolestanol (5a-Colestan-3aol $\left.-\mathrm{C}_{27} \Delta^{0}\right)$ apresentaram concentrações entre: 0,1 $\mu \mathrm{g} / \mathrm{g}$ (estação 34) - 2,8 $\mu \mathrm{g} / \mathrm{g}$ (estação 26); e 0,3 $\mu \mathrm{g} / \mathrm{g}$ (estação 34) - 1,5 $\mu \mathrm{g} / \mathrm{g}$ (estação 2), respectivamente. O 24-etilcoprostanol variou entre $0,5 \mu \mathrm{g} / \mathrm{g}$ (estação 34) - 6,2 $\mu \mathrm{g} / \mathrm{g}$ (estação 2). Dessa forma, pode-se observar que a estação 2 apresentou os níveis mais elevados de indicadores antrópicos, que pode estar relacionado a proximidade desta à desembocadura de esgotos domésticos (Jesus et al., 2004).

Esses valores podem ser considerados intermediários se comparados com as observações apresentadas na Tabela 3.

Tabela 3 - Concentração máxima de coprostanol ao longo de 3 localidades brasileiras.

\begin{tabular}{c|c|c|c}
\hline Região & coprostanol $(\boldsymbol{\mu g} / \mathrm{g})$ & colestanol $(\boldsymbol{\mu} \mathbf{g} \mathbf{g})$ & Referência \\
\hline $\begin{array}{c}\text { Baia de Guanabara } \\
\text { (RJ) }\end{array}$ & 40,0 & 10,9 & Carreira et al., 2004. \\
$\begin{array}{c}\text { Baía de Sepetiba (RJ) } \\
\text { Santa Catarina }\end{array}$ & 0,42 & -- & Carreira et al., 2009. \\
(manguezal impactado) & 2,8 & -- & Mater et al., 2004 \\
\hline
\end{tabular}

Uma ferramenta complementar na caracterização de fontes de esgoto é a utilização de alguns índices diagnósticos, onde estes podem ser calculados a partir das concentrações dos marcadores moleculares citados por Grimalt et al. (1990). Então, para o presente trabalho foram utilizados os índices: coprostanol/(colestanol+coprostanol), que pode ser representado como $5 \beta /(5 \alpha+5 \beta)$ estanol; e colesterol/ (colestanol+colesterol), como sendo $\Delta_{5} / 5 \alpha+\Delta_{5}$.

Os valores encontrados para o $5 \beta /(5 \alpha+5 \beta)$ estanol variaram entre 0,6 (estação 21) e 1,0 (estação 2). Já o $\Delta_{5} / 5 \alpha+\Delta_{5}$ pôde ser utilizado em um número maior de estações, sendo que os seus valores variaram entre 0,4 (estação 15) e 1,0 (estações 2, 4, 6, 7, 23 e 31$)$.

Valores para $5 \beta /(5 \alpha+5 \beta)$ estanol maiores do que 0,7 indicam áreas contaminadas, enquanto que valores entre 0,3 e 0,7 indicam regiões de contaminação intermediária (Grimalt et al., 1990). Dessa forma, os resultados encontrados para a Baía de Vitória podem classificá-la como uma região de contaminação intermediária, já o único valor acima de 0,7 foi observado na estação 2, que, como citado anteriormente, se encontra próxima a desembocadura de esgotamento sanitário.

Além da poluição oriunda de esgotos domésticos, caracterizada pelos esteróis, a presença de hidrocarbonetos alifáticos e da Mistura Complexa Não Resolvida (Unresolved Complex Mixture UCM) nos cromatogramas pode caracterizar material antrópico de origem petrogênica (Volkman et al., 1992). Hidrocarbonetos apresentam múltiplas fontes com relação à sua origem, podendo prover do aporte de esgotos domésticos e industriais, atividades portuárias, acidentes envolvendo petroleiros, outras fontes de petróleo, além das fontes naturais (Hostettler \& Kvenvolden, 1994; Dachs et al., 1999; Medeiros \& Bícego, 2004; Medeiros et al., 2005; Oliveira \& Madureira, 2011).

A presença de UCM foi observada nas estações localizadas na porção sudoeste do sistema, nas proximidades da área urbanizada. Esta região também apresentou os menores valores de IPC n-alcanos , sendo o IPC em torno de 1 indicativo da presença de hidrocarbonetos de petróleo e de matéria orgânica altamente degradada (Medeiros \& Bícego, 2004; Medeiros et al., 2005; Oliveira \& Madureira, 2011).

Outros tipos de hidrocarbonetos também são utilizados para caracterizar material petrogênico, são os hidrocarbonetos cíclicos do tipo hopanóides ou pentacíclicos triterpenóides, identificados através do íon base $\mathrm{m} / \mathrm{z}$ 191. A presença de hopanos com cadeia carbônica entre $C_{27}$ até $C_{35}$ com a configuração $17 \alpha(H), 21 \beta(H)$ são considerados biomarcadores específicos de petróleo (Hostettler \& Kvenvolden, 1994; Payet et al., 1999; Medeiros \& Bícego, 2004; Oliveira \& Madureira, 2011).

Neste trabalho foi observada a presença de alguns destes hopanóides, como o $17 \alpha(\mathrm{H}), 21 \beta(\mathrm{H})$ 30-norhopano, com concentrações variando entre $0,06 \mu \mathrm{g} / \mathrm{g}$ (estação 1) - 3,4 $\mu \mathrm{g} / \mathrm{g}$ (estação 9). O composto $17 \alpha(\mathrm{H}), 21 \beta(\mathrm{H})$-hopano, apresentou níveis entre $0,06 \mu \mathrm{g} / \mathrm{g}$ (estação 1) - 2,5 $\mu \mathrm{g} / \mathrm{g}$ (estação 9); já os compostos $17 \alpha(\mathrm{H}), 21 \beta(\mathrm{H})$-30-homohopano(22S) e $17 \alpha(H), 21 \beta(H)-30$-homohopano(22R) apresentaram $0,2 \mu \mathrm{g} / \mathrm{g}$ (estação 16) - 2,8 $\mu \mathrm{g} / \mathrm{g}$ (estação 21) e 0,2 $\mu \mathrm{g} / \mathrm{g}$ (estação 8 ) - 2,3 $\mu \mathrm{g} / \mathrm{g}$ (estação 11), respectivamente. As estações que apresentaram as maiores concentrações $(9,21$ e 11) estão localizadas próximas à margem, zonas preferenciais de sedimentação (Veronez Júnior et al., 2009) e próximas ao esgotamento sanitário (Jesus et al., 2004).

Valores dos hopanóides foram relativamente elevados quando comparados com os resultados encontrados de Medeiros e Bícego (2004) na Baía de Santos (SP), que observaram para os compostos apresentados anteriormente, concentrações máximas de: $0,59 \mu \mathrm{g} / \mathrm{g}(17 \alpha(\mathrm{H}), 21 \beta(\mathrm{H})-30$-norhopano); $0,60 \mu \mathrm{g} / \mathrm{g}$

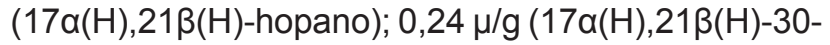
homohopano(22S)); e $0,18 \mu \mathrm{g} / \mathrm{g}(17 \alpha(\mathrm{H}), 21 \beta(\mathrm{H})-30$ homohopano(22R)). Uma possível explicação seria pelo fato desse ambiente apresentar sedimentos mais arenosos do que a Baía de Vitória, e como esses contaminantes se correlacionam preferencialmente com sedimento mais lamoso, eles podem ter se acumulado mais no sedimento do presente estudo.

O $17 \alpha(\mathrm{H}), 21 \beta(\mathrm{H})$-hopano foi o único hopano que apresentou correlação correlação positiva com 
menores granulometrias, e correlação negativa com maiores granulometrias conforme pode ser observado na Tabela 4.

Tabela 4 - Relação entre a granulometria e o composto $17 \alpha(H), 21 \beta(H)$-hopano (Correlação de Spearman, $P<0,05$ ).

\begin{tabular}{c|c}
\hline $\begin{array}{c}\text { Granulometria } \\
\text { (fi) }\end{array}$ & $\begin{array}{c}\text { Rho } \\
(\boldsymbol{\rho})\end{array}$ \\
\hline 22,5 & 0,5981 \\
31,5 & 0,5714 \\
15,62 & 5659 \\
11,25 & 0,517 \\
45 & 0,5042 \\
250 & -5338 \\
500 & $-0,5634$ \\
355 & $-0,5992$ \\
\hline
\end{tabular}

Dessa forma, as estações que apresentaram maiores concentrações destes compostos coincidem com aquelas com elevada porcentagem de lama.

Medeiros e colaboradores (2005), em um estudo realizado no Estuário da Lagoa dos Patos, encontraram valores de hopanos totais entre $0,017 \mu \mathrm{g} / \mathrm{g}$ e 4,30 $\mu \mathrm{g} / \mathrm{g}$, apresentando resultados mais elevados quando comparados ao presente estudo e com a Baía de Santos, sendo que ambas as regiões se caracterizam por elevada pressão antrópica.

Jovančićević et al. (2008) e Scholz-Böttcher et al. (2008), observaram em seus estudos a presença de hopanos $17 \alpha(H), 21 \beta(H)-\left(C_{29}-C_{30}\right)$ em amostras contaminadas por óleo que sofreram degradação microbiológica, e no sedimento superficial de uma região do Golfo do México, marcada por atividades antrópicas. Dessa forma, a presença destes biomarcadores de petróleo pode ser justificada pela intensa atividade portuária desenvolvida na região, além do despejo de efluentes industriais. O que também vai de acordo com as características observadas no trabalho realizado por Oliveira e Madureira (2011), em 3 regiões portuárias (SC), encontrando a presença destes compostos.

Apesar de o sistema estuarino da Baía de Vitória ser caracterizado por intensa atividade antrópica, a contribuição dos marcadores antrópicos representou uma pequena fração dos lipídeos totais observados. Sendo a contribuição mais significativa na porção sul da baía, que se caracteriza como uma região de intensa ocupação antrópica, além de ser fortemente influenciado pelas correntes de maré enchente (Saldanha, 2007), que pode influenciar nesta distribuição através da entrada de contaminantes provenientes de regiões portuárias além de esgotos domésticos.
Estas características evidenciam a presença das atividades antrópicas na região, apesar da baixa contribuição relativa em relação ao total de lipídeos caracterizados. Assim, o fato de o estuário da Baía de Vitória ser caracterizado como um ambiente com níveis intermediários de poluição, baseado nos presentes dados quando comparados com outros estuários urbanizados, pode ser justificado pela intensa e complexa hidrodinâmica local (Saldanha, 2007; Veronez Júnior et al., 2009), e pelo intenso aporte de sedimentos carreados pelo rio SMV, que ocorre, provavelmente, em função do desmatamento realizado tanto nas áreas de manguezais, quanto na vegetação que circunda a bacia hidrográfica, favorecendo a presença de compostos de origem terrestre (Saldanha, 2007).

A presença dos biomarcadores de petróleo (hopanóides) e da UCM no sedimento da Baía de Vitória pode ser justificada pela presença do Porto de Vitória, que sem encontra em uma região à jusante da área de estudo. Nesta região, também pode ser observado um elevado aporte de esgoto e águas residuais, devido à proximidade com a área urbana. Alguns autores (Medeiros \& Bícego, 2004; Medeiros et al., 2005; Oliveira \& Madureira, 2011), observaram a presença de aporte petrogênico em áreas com as mesmas características que as descritas para o presente estudo. O fato de o sistema estuarino da Baía de Vitória ser influenciado pela ação da maré, conforme citado anteriormente, justifica a presença desses compostos e características no sedimento amostrado.

\section{CONCLUSÕES}

O sistema estuarino da Baía de Vitória é marcado pela influência de múltiplas fontes de MO. Dessa forma, baseado nas observações dos resultados geoquímicos e dos índices calculados para AG (RTAe IPC) e para os $n$-alcanos (IPC e ACL), pôde-se perceber que a contribuição principal de material tem origem terrestre. $\mathrm{O}$ que pode ser evidenciado também pela elevada concentração dos pentacíclicos triterpenóides: $\beta$-amerina, taraxerol, germanicol, lupeol e $\alpha$-amerina, sendo a $\beta$-amerina o composto dominante. Estes podem indicar a contribuição do manguezal, além de outros tipos de vegetação terrestre, para a região. A razão $\mathrm{C} / \mathrm{N}$ também apresenta dados que reforça a ideia da influência terrestre, porém fica claro que há outras fontes naturais de material para a baía de Vitória, como o aporte aquático. Este, por sua vez, pôde ser caracterizado pela presença de AG de baixo peso molecular, ramificados e insaturados, além da presença de esteróis $\mathrm{C}_{27}$ e $\mathrm{C}_{28}$, sendo o colesterol o composto dominante dentre estes esteróis, encontrado 
em abundância em organismos marinhos, como o copépoda (zooplâncton dominante na região).

No presente trabalho também foi observado indícios de aporte antrópico para a região (esgoto e óleo fóssil), em função da intensa urbanização no entorno da baía, evidenciada pelos hopanóides (aporte petrogênico) e por esteróis fecais, apresentando o coprostanol como o mais abundante. Foi calculado índices para verificar o nível de contaminação fecal para o ambiente, onde o $5 \beta /(5 \alpha+5 \beta)$ estanol e o $\Delta_{5} / 5 \alpha+\Delta_{5}$, apresentaram níveis intermediários de poluição.

\section{AGRADECIMENTOS}

Agradecemos ao Fundo de Apoio a Ciência e Tecnologia (FACITEC) pelo financiamento do projeto, à Agência Nacional de Petróleo (ANP), ao Laboratório de Geoquímica da Matéria Orgânica da UFF e ao Laboratório de Oceanografia Geológica da UFES.

\section{REFERÊNCIAS}

Abu Bakar, N.; Tay, K. S.; Omar, N. Y. M. J.; Abas, M. R. B. \& Simoneit, B. R. T. 2011. The geochemistry of aliphatic and polar organic tracers in sediments from Lake Bera, Malaysia. Applied Geochemistry, 26: $1433-1445$.

Bourbonniere, R. A. \& Meyers, P. A. 1996. Sedimentary geolipid records of historical changes in the watersheds and productivities of Lakes Ontario and Erie. Limnology and Oceanography, 41(2): $352-359$.

Canuel, E. A. 2001. Relations between river flow, primary production and fatty acid compositon of particulate organic matter in San Francisco and Chesapeake Bays: a multivariate approach. Organic Geochemistry, 32: $563-583$.

Canuel, E. A. \& Martens, C.S., 1993. Seasonal variations in the sources and alteration of organic matter associated with recently-deposited sediments. Organic Geochemistry, 20: 563-577.

Carreira, R.; Wagner, A. L. R. \& Readman, J. W. 2004. Sterols as markers of sewage contamination in a tropical urban estuary (Guanabara Bay, Brazil): space-time variations. Estuarine, Coastal and Shelf Science, 60: $587-598$.

Carreira, R. S.; Ribeiro, P. V. \& Silva, C. E. M. 2009. Hidrocarbonetos e esteróis como indicadores de fontes e destino de matéria orgânica em sedimentos da Baía de Sepetiba, Rio de Janeiro. Química Nova, 32 (7): 1805 - 1811.

Dachs, J.; Bayona, J. M.; Fillaux, J.; Saliot, A. \&
Albaigés, J. 1999. Evaluation of anthropogenic \& biogenic inputs into the western Mediterranean using molecular markers. Marine Chemistry, 60: $195-210$.

Dodd, R. S.; Rafii, Z. A.; Fromard, F. \& Blasco, F. 1995. Biodiversity among west African Rhizophora: foliar wax chemistry. Biochemical Systematics and Ecology, 23 (7/8): 859 - 868.

Dodd, R. S.; Rafii, Z. A.; Fromard, F. \& Blasco, F. 1998. Evolutionary diversity among Atlantic coast mangroves. Acta Oecologica, 19 (3): $323-330$.

Elliott, M. \& McLusky, D. S. 2002. The Need for Definitions in Understanding Estuaries. Estuarine, Coastal and Shelf Science, 55: $815-827$.

Grilo, C. F.; Neto, R. R.; Vicente, M. A.; Castro, E. V. R. de; Figueira, R. C. L. \& Carreira, R. S. 2013. Evaluation of the influence of urbanization processes using mangrove and fecal markers in recent organic matter in a tropical tidal flat estuary. Applied Geochemistry, 38: 82 - 91.

Grimalt, J. O.; Fernández, P.; Bayona, J. M. \& Albagés, J. 1990. Assessment of fecal sterols and ketones as indicators of Urban Sewage Inputs to Coastal Waters. Environ. Sci. Technol., 24 (3): 357 - 363.

Hopkinson, C. S.; Buffam, I.; Hobbie, J.; Vallino, J.; Perdue, M.; Eversmeyer, B.; Prahl, F.; Covert, J.; Hodson, R.; Moram, M. A.; Smith, E.; Baross, J.; Crump, B.; Findlay, S. \& Foreman, K. 1998. Terrestrial inputs of organic matter to coastal ecosystems: An intercomparison of chemical characteristics and bioavailability. Biogeochemistry, 43: $211-234$.

Hostettler, F. D. \& Kvenvolden, K. A. 1994. Geochemical changes in crude oil spilled the Exxon Valdez supertanker into Prince William Sound, Alaska. Organic Geochemistry, 21(8/9): $927-936$.

Isaaks, E. H. \& Srivastava, R. M. 1989. Applied geostatistics. New York: Oxford University.

Jaffé, R.; Wolff, G. A.; Cabrera, A. C. \& Chitty, H. C. 1995. The biogeochemistry of lipids in rivers of the Orinoco Basin. Geochimica et Cosmochimica Acta, 59 (21): 4507 - 4522.

Jaffé, R.; Mead, R.; Hernandez, M. E.; Peralba, M. C. \& Diguida, O. A. 2001. Origin and transport of sedimetary organic matter in two subtropical estuaries: a comparative, biomarker-based study. Organic Geochemistry, 32: 507-526.

Jaffé, R.; Rushdi, A. I.; Medeiros, P. M. \& Simoneit, B. R. T. 2006. Natural product biomarkers as indicators of sources \& transport of sedimentary organic matter in a subtropical river. Chemosphere, 64: 1870-1884.

Jeng, W. L. 2006. Higher plant n-alkane average chain length as a indicator of petrogenic hydrocarbon contamination of marine sediments. Marine 
Chemistry, 102: $242-251$.

Jesus, H. C.; Costa, E. A.; Mendonça, A. S. F. \& Zandonade, E. 2004. Distribuição de Metais Pesados no Sistema Estuarino da llha de VitóriaES. Quimica Nova, 27 (3): 378 - 386.

Johnston, K.; Ver Hoef, J. M.; Krivoruchko, K. \& Lucas, N. 2001. Using ArcGIS TM Geostatistical Analyst. Copyright.

Jovančićević, B.; Antieć, M.; Pavlović, I.; Vrvić, M.; Beskoski, V.; Kronimus, A. \& Schwarzbauer, J. 2008. Transformation of petroleum saturated hydrocarbons during soil bioremediation experiments. Water Air Soil Pollution, 190: 299 $-307$.

Killops, S. D. \& Frewin, N. L. 1994. Triterpenoid diagenesis and cuticular preservation. Organic Geochemistry, 21(12): 1193 - 1209.

Koch, B. P.; Rullkötter, J. \& LARA, R. J. 2003. Evaluation of triterpenols and sterols as organic matter biomarkers in a mangrove ecosystem in northern Brazil. Wetlands Ecology and Management, 11: $257-263$.

Koch, B. P.; Harder, J.; Lara, R. J. \& Kattner, G. 2005. The effect of seletive microbial degradation on the composition of mangrove derived pentacyclic triterpenols in surfece sediments. Organic Geochemistry, 36: $273-285$.

Kristensen, E.; Bouillon, S.; Dittmar, T. \& Marchand, C. 2008. Organic carbon dynamics in mangrove ecossistems: A review. Aquatic Botany, 89: 201 $-2019$.

Leeming, R.; Ball, A.; Ashbolt, N. \& Nichols, P. 1996. Using faecal sterols from humans and animals to distinguish faecal pollution in receiving waters. Water Resolution, 30 (12): 2893 - 2900.

Lima, T.M.J. 2011. Efeitos da frequência de inundação na estrutura do manguezal da Baía de Vitória, Vitória-ES. Dissertação (Mestrado em Oceanografia Ambiental) - Universidade Federal do Espírito Santo.

Mackenzie, A. S.; Brassel, S. C.; Eglinton, G. \& Maxwell, J. R. 1982. Chemical Fossils: The Geological Fate of Steroids. Science, 217: $491-504$.

Mahapatra, D. M.; Chanakya, H. N. \& Ramachandra, T. V. 2011. C:N ratio of sediments in a sewage fed urban lake. International Journal of Geology, 3(5): $86-92$.

Mannino, A. \& Harvey, H. R. 1999. Lipid composition in particulate and dissolved organic matter in the Delaware Estuary: Sources and diagenetic patterns. Geochimica et Cosmochimica Acta, 63 (15): $2219-2235$.

Mater, L.; Alexandre, M. R.; Hansel, F. A. \& Madureira, L. A. S. 2004. Assessment of lipid compounds \& phosphorus in mangrove sediments of Santa
Catarina Island, SC, Brazil. Journal of the Brazilian Chemical Society, 15 (5): 725 - 734.

McCallister, S. L.; Bauer, J. E.; Ducklow, H. W. \& Canuel, E. A. 2006. Sources of estuarine dissolved \& particulate organic matter: A multi-tracer approach. Organic Geochemistry, 37: 454-468.

Medeiros, P. M. \& Bícego, M. C. 2004. Investigation of natural and anthropogenic hydrocarbon inputs in sediments using geochemical markers in Santos, SP-Brazil. Marine Pollution Bulletin, 49: 761 - 769.

Medeiros, P. M.; Bícego, M. C.; Castelao, R. M.; Rosso, C. D.; Fillman, G. \& Zamboni, A. J. 2005. Natural and anthropogenic hydrocarbon inputs to sediments of Patos Lagoon Estuary, Brazil. Environmental International, 31: 77 - 87.

Meyers, P. A. 1994. Preservation of elemental and isotopic source identification of sedimentary organic matter. Chemical Geology, 144: 289 - 302.

Meziane, T. \& Tsuchiya, M. 2000. Fatty acids as tracers of organic matter in the sediment \& food web of a mangrove/intertidal flat ecosystem, Okinawa, Japan. Marine Ecology Progress Series, 200: $49-57$.

Moberg, F. \& Rönnbäck, P. 2003. Ecosystem services of the tropical seascape: interactions, substitutions and restoration. Ocean and Coastal Management, 46: 27-46.

Nalesso, R. C.; Joyeux, J. C.; Quintana, C. O.; Torezani, E. \& Otegui, A. C. P. 2005. Soft-bottom macrobenthic communities of the Vitoria Bay Estuarine System, South-Eastern Brazil. Brazilian Journal of Oceanography, 53(1/2): $23-38$.

Oliveira, C. R. de \& Madureira, L. A. dos S. 2011. Assessment and sources of nonaromatic hydrocarbons in surface sediments of three harbors in Santa Catarina State, Brazil. Environ Monit Assess, 173: 91 - 105.

Otto, A. \& Simpson, M. J. 2005. Degradation and preservation of vascular plant-derived biomarkers in grassland and forest oils from Western Canada. Biogeochemistry, 74: $377-409$.

Payet, C.; Bryselbout, C.; Morel, J.-L.; Lichtfouse, E. 1999. Fossil fuel in sewage sludges: environmental significance. Naturwissenschaften, 86: $484-488$.

Rontani, J. F. \& Volkman, J. K. 2005. Lipid characterization of coastal hypersaline cyanobacterial mats from the Camargue (France). Organic Geochemistry, 36: $251-272$.

Saldanha, J. C. S. 2007. Análise da influência do rio Santa Maria da Vitória na Baía de Vitória, através da modelagem computacional: uma contribuição ao processo de enquadramento. Dissertação de Mestrado (Ciências em Engenharia Ambiental). Universidade Federal do Espírito Santo. Vitória.

Silva, C. A.; Oliveira, C. R.; Oliveira, I. R. W. Z.; 
Madureira, L. A. S. 2008. Distribution of Lipid Compounds in Sediments from Conceição Lagoon, Santa Catarina Island, Brazil. Journal of the Brazilian Chemistry Society, 19 (8): 1513 - 1522.

Scholz-Böttcher, B. M.; Ahlf, S.; Várquez-Gutiérrez, F. \& Rullkötter, J. 2008. Sources of hydrocarbon pollution in surface sediments of the Campeche Sound, Gulf of Mexico, revealed by biomarker analysis. Organic Geochemistry, 39: $1104-1108$.

Simoneit, B. R. T. 1986. Cyclic terpenoids of the geosphere. In: Jhons, R. B. Biological Markers in the Sedimentary Record. Elsevier,2: 43 - 99.

Sterza, J. N. \& Fernandes, L. L. 2006. Zooplancton community of the Vitoria Bay Estuarine System (southeastern Brazil). Characterization of threeyears study. Brazilian Journal of Oceanography, 54(2/3): $95-105$.

Takada, H., Satoh, F., Bothner, M.H., Tripp, B.W., Johnson, C.G. \& Farrington, J.W. 1997. Anthropogenic molecular markers: tools to identify the sources and transport pathways of pollutants. In: Eganhouse, R.P. (Ed.), Molecular Markers in Environmental Geochemistry. ACS Symposium Series n. 671, American Chemical Society. Washington, 178 - 195.

Ten Haven, H. L. \& Rullkötter, J. 1988. The diagenetic fate of taraxer-14-ene and oleanene isomers. Geochimica et Cosmochimica acta, 52: 2543 $-2548$.

Ten Haven, H. L.; Peakman, T. M. \& Rullkötter, J. 1992a. $\Delta 2$ - Triterpenes: Early intermediates in the diagenesis of terrigenous triterpenoids. Geochimica et Cosmochimica Acta, 56: 1993 $-2000$.

Ten Haven, H. L.; Peakman, T. M. \& Rullkötter, J. 1992b. Early diagenetic transformation of higherplant triterpenoids in deep-sea sediments from Baffin Bay. Geochimica et Cosmochimica Acta, 56: $2001-2024$.
Veronez Júnior, P.; Bastos, A. C. \& Quaresma, V. S. 2009. Morfologia e distribuição sedimentar em um sistema estuarino tropical: Baía de Vitória, ES. Revista Brasileira de Geofísica, 27(4): 609 - 624.

Versteegh, G. J. M.; Schefuß, E.; Dupont, I.; Marret, F.; Damsté, J. S. S. \& Jansen, J. H. F. 2004. Taraxerol and Rhizophora pollen as proxies for tracking past mangrove ecosystems. Geochimica et Cosmochimica Acta, 64(3): 411-422.

Volkman, J. K. 1986. A review of sterol markers for marine and terrigenous organic matter. Organic Geochemistry, 9 (2): $83-99$.

Volkman, J. K.; Holdsworth, D. G.; Neil, G. P. \& Bavor Jr., H. J. 1992. Identification of natural, anthropogenic and petroleum hydrocarbons in aquatic sediments. The Science of the Total Environment, 112: 203-219.

Volkman, J. K.; Barret, S. M.; Blackburn, S. I.; Mansour, M. P.; Sikes, E. L. \& Gelin, F. 1998. Microalgal biomarkers: A review of recent research developments. Organic Geochemistry, 29(5-7): 1163 - 1179.

Volkman, J. K. 2003. Sterols in Microorganisms. Appl Microbiol Biotechnol, 60: 495 - 506.

Volkman, J. K.; Revill, A. T.; Bonham, P. I. \& Clementson, L. A. 2007. Sources of organic matter in sediments from the Ord River in tropical northern Australia. Organic Geochemistry, 38: 1039 - 1060.

Xu, Y.; Mead, R. N. \& Jaffé, R. 2006. A molecular marker-based assessment of sedimentary organic matter sources and distributions in Florida Bay. Hydrobiologia, 569: 179 - 192.

Submetido: Agosto/2013

Revisado: Novembro/2015 Aceito: Maio/2016 